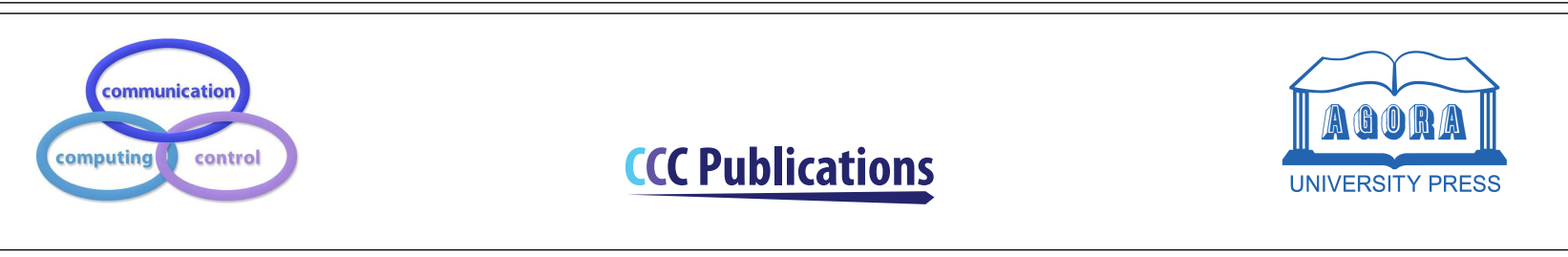

\title{
Bearing Fault Diagnosis Method Based on EEMD and LSTM
}

\author{
P. Zou, B. Hou, L. Jiang, Z. Zhang
}

\section{Ping Zou}

1. School of Economics and Management, Beijing Jiaotong University, China

No.3 Shangyuancun Haidian District Beijing 100044 P. R. China 18113038@bjtu.edu.cn

2. Beijing Aerospace Smart Manufacturing Technology Development CO., Ltd, China

49 Badachu Road, Shijingshan Dist. Beijing 100144 P. R. China ziapple@126.com

\section{Baocun Hou}

Beijing Aerospace Smart Manufacturing Technology Development CO., Ltd, China

49 Badachu Road, Shijingshan Dist. Beijing 100144 P. R. China

houbaocun@casicloud.cn

\section{Lei Jiang}

Railway Information Center, China

China National Railway Corporation,

Beijing 100844, China

ginajiang1984@163.com

\section{Zhenji Zhang*}

School of Economics and Management, Beijing Jiaotong University, China

No.3 Shangyuancun Haidian District Beijing 100044 P. R. China

*Corresponding author: zhangzhenji@bjtu.edu.cn

\begin{abstract}
The condition monitoring and fault detection of rolling bearing are of great significance to ensure the safe and reliable operation of rotating machinery system.In the past few years, deep neural network (DNN) has been recognized as an effective tool to detect rolling bearing faults. However, It is too complex to directly feed the original vibration signal to the DNN neural network, and the accuracy of fault identification is not high. By using the signal preprocessing technology, the original signal can be effectively removed and preprocessed without losing the key diagnosis information. In this paper, a new EEMD-LSTM bearing fault diagnosis method is proposed, which combines the signal preprocessing technology with the EEMD method that can get clear fault feature signals, and LSTM technology to extract fault features automatically that improves the efficiency of fault feature extraction. In the case of small sample size, this method can significantly improve the accuracy of fault diagnosis.
\end{abstract}

Keywords: fault diagnosis, EEMD, LSTM, motor bearing. 


\section{Introduction}

Rotating machinery is widely used in modern manufacturing, aerospace, vehicles, wind turbines and other fields. Fault diagnosis of key components of rotating machinery, especially bearings, plays an important role in the reliability and safety of modern industrial system [19]. Research on fault diagnosis methods of bearings is of great significance to maintain technical status of equipment and equipment and extend the service time.In the past few decades, major fault diagnosis technology based on vibration signal analysis can be divided into signal processing method [21], intelligent diagnosis method [22] and remaining service life prediction method [24]. The signal preprocessing method is very important for bearing fault feature extraction and fault recognition accuracy.On the one hand, the bearing vibration signal is often disturbed by the external environment noise, which makes the signalto-noise ratio low.On the other hand, there are many kinds of bearing parts, the vibration signal measured shows obvious nonlinearity, and the vibration caused by large-scale transient fluctuation of load has strong non-stationary.Therefore, it is necessary to preprocess the acquired original signal to enhance the feature information, so as to obtain better diagnosis results.

EMD (empirical mode decomposition) is a time-frequency signal decomposition tool proposed by N.E. Huang for analyzing non-stationary and non-linear signals [15]. It is a typical signal processing method and widely used in the field of mechanical fault diagnosis [13]. EMD can decompose complex signals into sum of a series of intrinsic mode function (IMF), and each IMF component represents an inherent vibration mode of signals, including different characteristic time scales, so that the signal characteristics are displayed at different resolutions.Because the frequency component of each IMF is not only related to the sampling frequency, but also changes with the change of the signal itself, EMD method is an adaptive signal decomposition method with high signal-to-noise ratio, which is very suitable for processing time-varying and non-stationary signals.However, mode mixing is the most significant disadvantage of EMD [25]. EEMD proposed by Z. Wu and N.E. Huang is an improved method [28], which solves the problem of mode mixing phenomenon by adding white noise into the original signal. The spectrum obtained by EEMD method reflects the bearing fault characteristics more accurately.Based on the EEMD method, scholars have made some achievements in the field of fault diagnosis.Yaguo Lei proposed a fault feature extraction method based on EEMD [17], which is applied to the early rub impact fault diagnosis of heavy oil catalytic cracking unit.In order to solve the problem of EMD mode mixing, Tong Wang proposed a noise aided data analysis method called set experience [26], and studied the influence of EMD and EEMD on time-frequency.The pattern recognition methods used in these studies are mainly based on the "shallow learning" algorithm, which has high requirements for signal acquisition and processing, limited expression ability for complex functions, and poor generalization ability of the model.

Deep neural network (DNN) originated from artificial neural network (ANN) [5], widely used in vision, speech recognition and natural language processing. Various deep learning algorithms, such as auto encoder, stack auto encoder [6], DBM and DBN [14], have also been successfully applied to fault diagnosis.Deep learning is a powerful feature learning ability, which can meet the requirements of adaptive feature extraction of mechanical fault diagnosis. Its application in the field of fault diagnosis can reduce the dependence on expert fault diagnosis experience and signal processing technology, and reduce the uncertainty introduced by traditional fault diagnosis methods due to artificial design and feature extraction.In addition, deep learning can learn the complex mapping relationship between monitoring data and fault state by building a deep model with multiple hidden layers and multi-level abstraction in the way of nonlinear mapping, which is very suitable for fault diagnosis in the context of complex data.

Convolution neural network $(\mathrm{CNN})$ and recurrent neural network $(\mathrm{RNN})$ are the typical methods of DNN.Most fault vibrations, such as flaking vibration, are periodic due to repeated impact. The amplitude and period depend on the bearing specifications and operating conditions. It is difficult for CNN to identify the continuous changes of these vibration characteristics. Therefore, CNN is not enough to diagnose continuous and periodic faults. Recursive neural network (RNN) architecture, especially longterm and short-term memory (LSTM) neural network and its variants, has been successfully applied in the fields of image subtitle, speech recognition, genome analysis and natural language processing [3]. LSTM is a kind of special RNN, which can extract the characteristic information contained in 
the data of sea going volume efficiently, and can learn the dynamic change of time series adaptively, and can solve the problem of gradient disappearance and gradient explosion in the process of long series training. The experiment shows that the accuracy is better than the conventional RNN model, and can effectively improve the diagnosis rate [16]. Zhao, R. obtains the long-term dependence of vibration signal through the LSTM model and models the sequence data, monitors the health status of the machine [32]. Xiang Xu uses the method based on LSTM to improve the accuracy of fault identification in power system [31].

This paper focuses on the intelligent fault diagnosis of the bearing of the rotating electrical machine by referring to the research progress of deep learning. According to the non-stationary characteristics of bearing vibration signal, EEMD method is combined with LSTM. EEMD is used as the preprocessor of vibration signal. IMF component with obvious fault feature information is selected according to the kurtosis of each component. Then, the information of IMF component is adaptively fused by LSTM and the feature is extracted from it to complete the intelligent classification and recognition of bearing state.At last, the method is compared and analyzed with other fault diagnosis methods by test data. The results show that the performance of the proposed method is far better than SVM and BPNN. Compared with emd-cnn, the method has higher accuracy and better stability.

\section{EEMD ensemble empirical mode decomposition method}

In recent years, empirical mode decomposition (EMD) is an effective method to deal with nonlinear and non-stationary signals. Its main advantage is to decompose complex signals into several intrinsic mode functions (IMF) arranged from high to low frequency, which overcomes the difficulty of selecting basis functions in wavelet transform, but it can not overcome the problem of modal aliasing caused by noise [29].In order to solve the mode aliasing problem, N.E Huang added the Gauss white noise assisted decomposition method on the basis of EMD, which is the ensemble empirical mode decomposition(EEMD). Its basic principle is to use the uniform distribution characteristics of Gauss white noise spectrum, add the white noise into the whole time-frequency distribution space, and take the "average" of the results of multiple decomposition. Finally, they are cancelled each other to promote anti aliasing decomposition and avoid modal aliasing. The flow of EEMD algorithm is shown in Figure 1. The steps of EEMD decomposition are as follows:

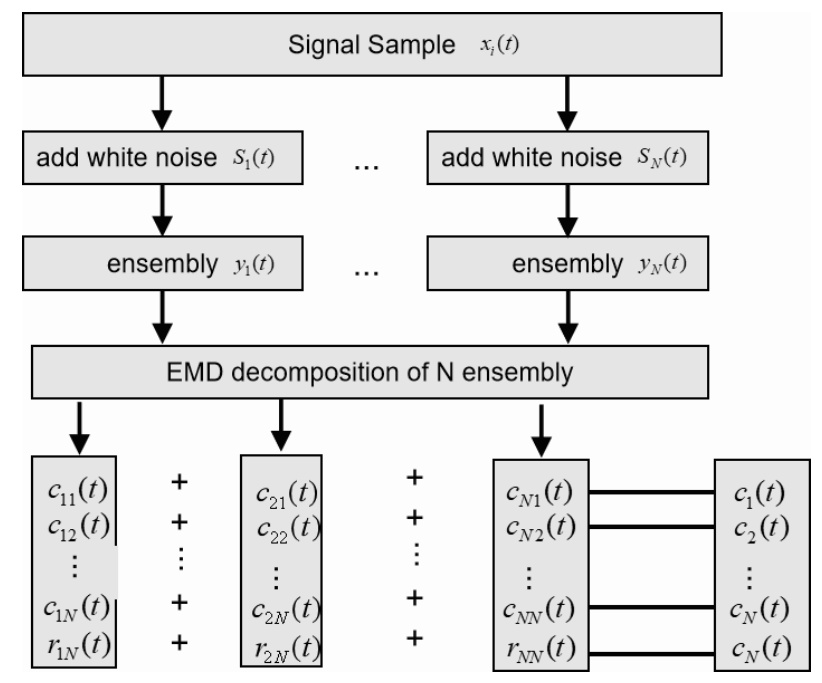

Figure 1: EEMD decomposition steps

(1) Add $n$ groups of different Gaussian white noise $s_{i}(t)$ into the original signal $x(t)$, so that the signal and noise become $\mathrm{n}$ populations

$$
y_{i}(t)=x(t)+s_{i}(t)
$$

(2) EMD decomposition of $\mathrm{N}$ populations with white noise is carried out to obtain the corresponding intrinsic modal function components (IMF) $c_{i j}(t)$ and remainder terms $r_{i}(t) . c_{i j}(t)$ represents the 
j-th IMF obtained by decomposition after the i-th white noise is added.

(3) The final decomposition result of EEMD is obtained by averaging the corresponding IMF in step 2

$$
c_{i}(t)=1 / N \sum_{i=1}^{N} c_{i j}(t)
$$

where $c_{j}(t)$ represents the j-th component obtained after EEMD decomposition of the original signal. Compared with one EMD decomposition, EEMD can eliminate mode aliasing, making the physical meaning of IMF clearer.

(4) IMF component selection: each modal function component decomposed $I M F_{i}$ is a narrow band signal from high frequency to low frequency. However, when EEMD noise reduction, IMF component should be properly selected to retain the most obvious IMF component of fault feature information. Kurtosis has nothing to do with bearing speed, size and load, etc. It is very sensitive to impact signal. It is particularly suitable for surface damage faults, especially early faults diagnosis. Through kurtosis analysis of $I M F_{i}$ components, IMF components with obvious fault feature information are selected, and the signal kurtosis formula is as follows:

$$
C_{q}=\frac{\frac{1}{N} \sum_{i=1}^{N}\left(\left|x_{i}\right|-\bar{x}\right)^{4}}{X_{r m s}^{4}}
$$

where $X_{r m s}$ represents the root mean square value of the signal and is an important index used to determine whether the operation state is normal in the mechanical fault. By kurtosis selection [18], the reserved component $I M F_{i}$ fault feature signal is more significant.

\section{$3 \quad$ LSTM related work}

\subsection{LSTM structure}

The fault signal component IMF extracted by EEMD usually has the characteristics of time cycle, and the fault features often have periodicity and repeatability in the time series. The long-term short-term memory (LSTM) recurrent neural network can remember the observation results of longterm sequence interval. LSTM adopts a network model which uses the form of similar list and has certain memory ability. Compared with the composition of general neural network, RNN will add an additional propagation matrix from T-1 time hidden layer to $t$ time hidden layer, as shown in Figure 2 below. For sample data with time series, it is necessary to remember the past information to predict the current state, so that the fault features in the sample can be effectively extracted. Long Short-term memory (LSTM) is a kind of time cycle neural network, which is specially designed to solve the long-term dependence problem of recurrent neural network(RNN) [1]. As shown in Figure 2 , LSTM is a chain form of repeated neural network modules.

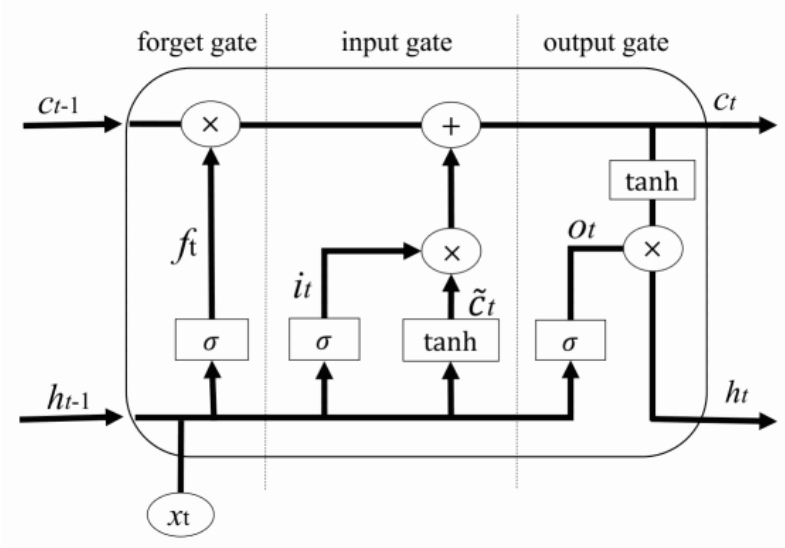

Figure 2: LSTM structure 
Forgetting gate, input gate and output gate are the core components of LSTM, which are composed of multiple feature maps. The forgetting gate determines how much front information passes, and the output results are as follows:

$$
f_{t}=\sigma\left(w_{f z} z_{t}+w_{h f} h_{t-1}+b_{f}\right)
$$

where $\sigma$ represents the activation function, $w$ represents the weight, $z t$ represents the current input, and $h_{t-1}$ is the input result of the previous neuron. $b_{f}$ represents offset.

The input gate determines which information can be retained. The calculation formula is as follows:

$$
\begin{gathered}
i_{t}=\sigma\left(w_{z i} z_{t}+w_{h i} h_{t-1}+b_{i}\right) \\
\tilde{c_{t}}=\tanh \left(w_{x c} z_{t}+w_{h c} h_{t-1}+b_{i}\right)
\end{gathered}
$$

The output gate determines which information can be output as a unit, and the calculation formula is as follows:

$$
\begin{gathered}
c_{t}=c_{t-1} f_{t}+i_{t} \tilde{c_{t}} \\
o_{t}=\sigma\left(w_{z o} z_{t}+w_{h o} h_{t-1}+b_{o}\right) \\
h_{t}=o_{t} \times \tanh \left(c_{t}\right)
\end{gathered}
$$

After the LSTM layer is the layer for classification, and the calculation formula is as follows:

$$
\operatorname{soft} \max \left(y_{i}\right)=\frac{e^{u_{i}}}{\sum e^{u_{i}}}
$$

\subsection{Training methods}

In order to minimize the training error, gradient descent method is used [3]. The main problem of gradient descent method in recurrent neural network (RNN) was first found in 1991, that is, the error gradient disappears exponentially with the time length between events [4]. When the LSTM block is set, the error is also calculated along with the back propagation, from output to every gate in the input stage until the value is filtered out.Therefore, the normal back transfer neural network is an effective method to train LSTM block to remember long-term values. The cross entropy is chosen as the cost function to express the error between the real value and the estimated value:

$$
L=-\sum x p(x) \ln ^{q(x)}
$$

where $p(x)$ represents the real classification result, and $q(x)$ is the classification result output by soft max.

Using a method for stochastic optimization(ADAM) to train LSTM and determine network parameters, that is, weight and deviation.Adam can dynamically adjust the learning rate of each parameter by using the first-order moment estimation (mean) and second-order moment estimation (variance) of the gradient, and has achieved success in optimizing the learning rate of LSTM.

\subsection{LSTM design}

LSTM has been widely used in natural language processing(NLP) field [2], which is suitable for dealing with and predicting important events with relatively long interval and delay in time series. At present, it has been proved that LSTM is an effective technology to solve the problem of long sequence dependence, and the general adaptability of this technology is very high. Bearing fault information is also time series data. The fault information is sliced according to time-domain dimension, and fixed slice length is used as time-series sample, which is also suitable for LSTM neural network.

The key parameter of LSTM is to select the time step, as shown in the figure below. The IMF component obtained from the EEMD decomposition of the signal sample $x_{i}(t)$ is taken as the time step, so that the LSTM network can remember the fault feature signal, which is conducive to improving the accuracy of fault diagnosis. The IMF component with obvious fault feature information is adaptively fused by LSTM, and the LSTM parameter obtained through training is the weight of each component fusion, among which the parameters of LSTM neuron of time step are shared. 


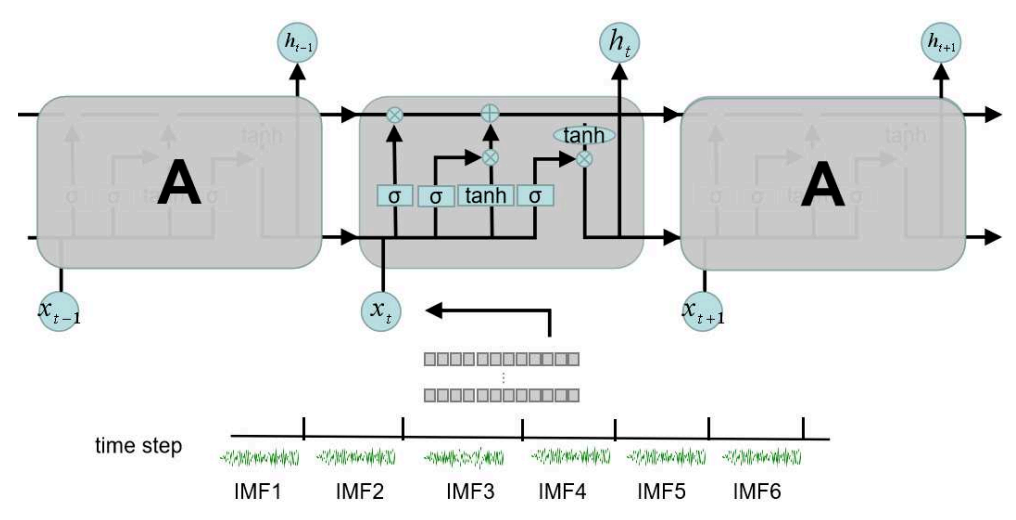

Figure 3: Time step design of LSTM

The performance of LSTM is related to the number of network layers and the size of output layer.First, the learning ability of LSTM is positively related to the number of network layers.To a certain extent, the deeper the network structure, the stronger the expression ability. Multi layer LSTM is to stack LSTM. Its advantage is that it can express features more abstractly at high level, reduce the number of neurons, increase recognition accuracy and reduce training time.If the structure of LSTM is too simple and the learning ability is poor, the information contained in the input cannot be extracted effectively. The deep structure of the network means that more training data is needed to train a large number of parameters, otherwise, over fitting and local optimization problems may occur. At the same time, due to the increasing complexity of the network, it is very time-consuming to train the model, which increases the difficulty of building a suitable and efficient model.Secondly, the output size of LSTM needs to be tested and determined according to some basic design principles.Generally speaking, the longer the time step is, the higher the output dimension of the LSTM is, and the more information it remembers [7], more data can be obtained and more information can be provided for the deeper level. More importantly, for mechanical fault diagnosis, it can better suppress high-frequency noise and improve the accuracy of fault identification.In addition, the output dimension of LSTM in the first layer is set to a large size to deepen the network and improve the expression ability of the network, so as to learn to obtain a good feature representation of the input signal.Although some basic principles of setting super parameters are mentioned above, a lot of debugging is still needed in the actual process to get the appropriate parameter value [30].

\section{Fault diagnosis method flow}

The bearing fault diagnosis based on EEMD and LSTM is divided into two main steps. The first step is signal preprocessing, which decomposes the sample signal into EEMD and filters the fault characteristic signal according to the kurtosis. The second step is to combine the sample signal preprocessing as input and provide it to the RNN neural network of LSTM. The flow is shown in Figure 4.

Step 1: signal preprocessing

(1) signal acquisition and division: obtain bearing vibration signal through acceleration sensor, divide it with equal length window, acquire sample signal, and more samples can be extracted from fixed length signal. Using this method, time-domain signal with length of $g$ can be divided into $n$ samples, in which the length of sample is moving distance of slice window $s$, forming $n$ samples with dimension length of $s$.

(2) EEMD decomposition of sample signal: EEMD decomposition of sample signal obtained from vibration signal slice is carried out to decompose non-stationary signal $x(t)$ into $m$ stationary signals with different characteristic scales (IMF1, IMF2,..., IMFM). Then by analyzing the kurtosis of IMF components, $k$ IMF components with obvious fault characteristic information are selected.

(3) data set merging and creation: stack $k$ IMF components with obvious fault characteristics into a multi-channel sample to form a matrix of $[k, s]$, perform the above operations on all sample signals, 


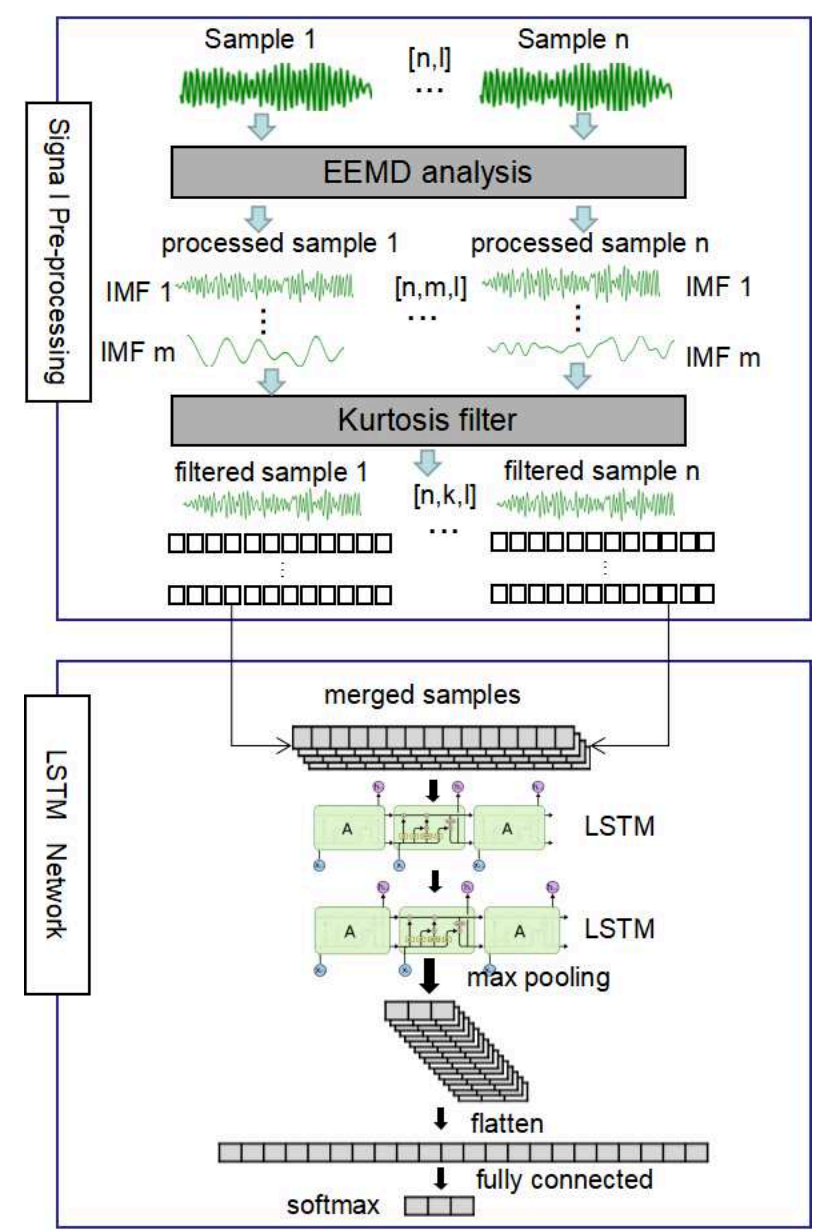

Figure 4: Fault diagnosis method flow based on EEMD and LSTM

merge and create a data set, form a sample data set of $[n, k, s]$ dimension, and divide the data set into training set and test set.

\section{Step 2: LSTM neural network training}

(1) LSTM design and training: design feasible LSTM in accordance with the design principles described in Section 3.3 and use the training set for training, debug the super parameters, reduce the network calculation through the pooling layer, increase the model generalization ability, use the full connection layer and classify the training results, and obtain a better performance LSTM model through network iterative training.

(2) Qualitative diagnosis of bearing fault: verify the validity of bearing fault diagnosis model based on EEMD and LSTM through test set.

\section{Test}

\subsection{Test description}

In this paper, PHM 2009 challenge data of Xichu university [33] is used as experimental data to analyze and verify the EEMD + LSTM method proposed in this paper. As shown in Figure 5 and Figure 6 below, the gearbox test bench includes a 2 horsepower motor (left), a torque sensor / encoder (middle), a dynamometer (right) and control electronic equipment. The test bearing is used to support the motor shaft. Each pair of meshing gears contains a spur gear and a helical gear. Acceleration data are measured near and far away from the motor bearing. Single point fault is introduced into the test bearing by EDM.

The experimental environment is shown in Table 1 below. The faults between 0.007 inch and 0.040 inch in diameter occur on the inner raceway, rolling element (i.e. ball) and outer raceway respectively, 


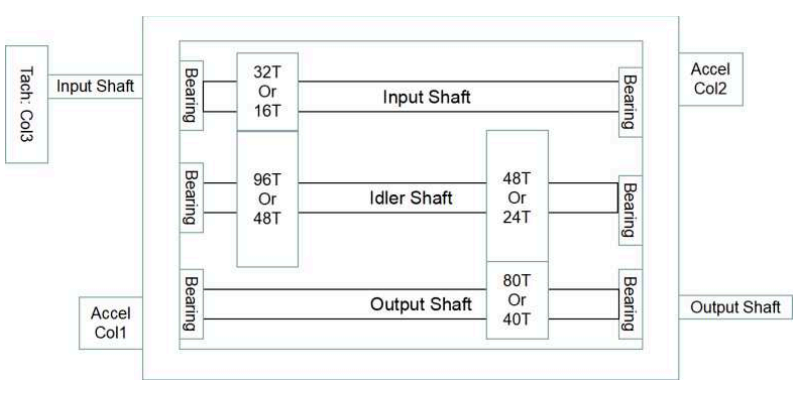

Figure 5: Structural diagram of gearbox (a)

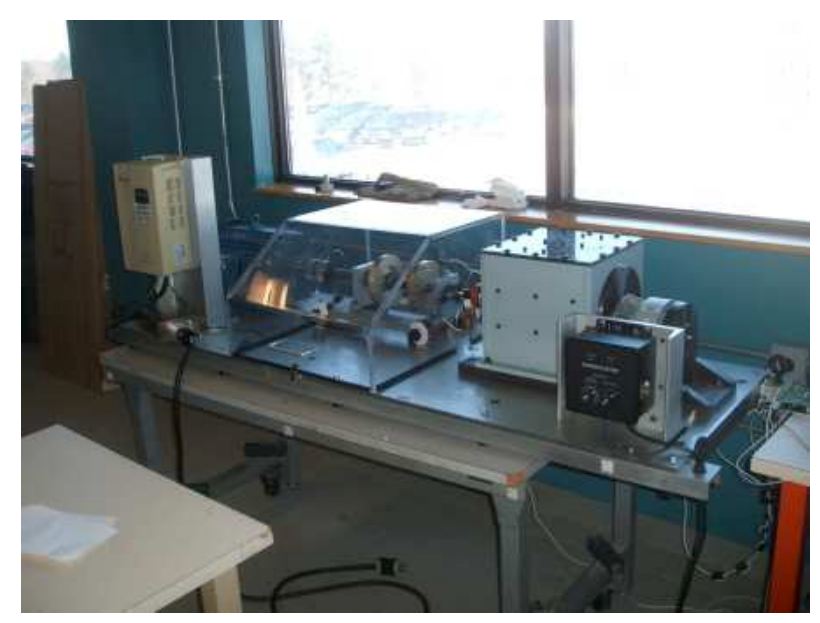

Figure 6: Structural diagram of gearbox (b)

and the vibration data of 0-3 horsepower (motor speed 1797-1720 RPM) motor load are recorded.

\subsection{Data description}

In this paper, $1797 / \mathrm{min}$, load $0,12 \mathrm{~K}$ sampling rate normal data and 10 sets of data for inner ring, sphere and outer ring under 0.007, 0.014 and 0.021 fault diameter conditions are used. Each group of sample signal contains 120000 sampling points (continuous acquisition for 10s), with an average of $12000 \times 60 \backslash 1797=400$ data points per cycle, forming $120000 \times 10 \backslash 400=3000$ samples. The IMF sample component of the vibration acceleration sample signal of a single motor rotating periodic bearing after passing through EEMD is shown in Figure 7.

Different IMF components contain different time scales, which can display the characteristics of signals at different resolutions, while the fault feature information generated by bearings is mainly contained in the first several IMF components. When the rolling bearing is damaged locally, the impact component will be produced. The impact component usually contains the fault feature information, so kurtosis is selected as the judgment standard of effective IMF. The kurtosis of each IMF component $\mathrm{f}$ is shown in Figure 8.

Generally, the higher the kurtosis is, the more impact components are contained in the signal. According to the kurtosis threshold, the six components with the largest kurtosis are selected as

Table 1: Description of experimental environment parameters

\begin{tabular}{cc}
\hline Experimental environment & Parameter value \\
\hline Approx. Motor Speed (RPM) & $1797 / \mathrm{min}, 1772 / \mathrm{min}, 1750 / \mathrm{min}, 1730 / \mathrm{min}$ \\
Sample rate & $12 \mathrm{k}, 48 \mathrm{~K}$ (Drive End Bearing Fault Data) \\
Motor Load (HP) & $0,1,2,3$ \\
Race fault diameter & $0.007,0.014,0.021$ \\
Ball failure diameter (ball) & $0.007,0.014,0.021$ \\
Diameter of outer ring failure & $0.007,0.014,0.021$ \\
\hline
\end{tabular}




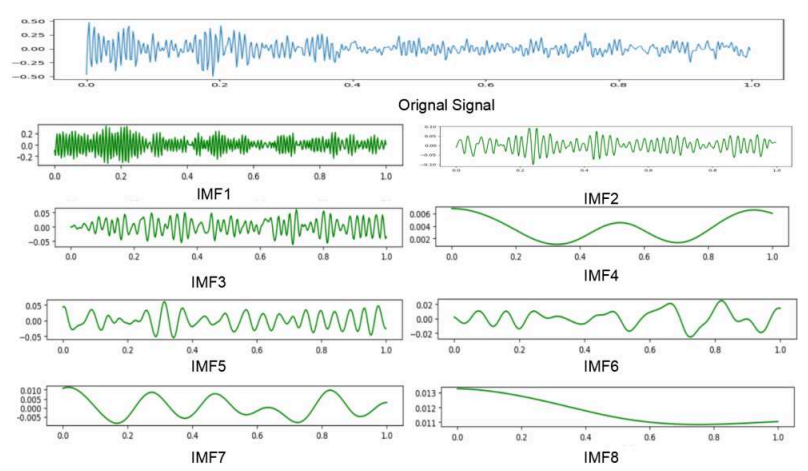

Figure 7: IMF component diagram after EEMD decomposition

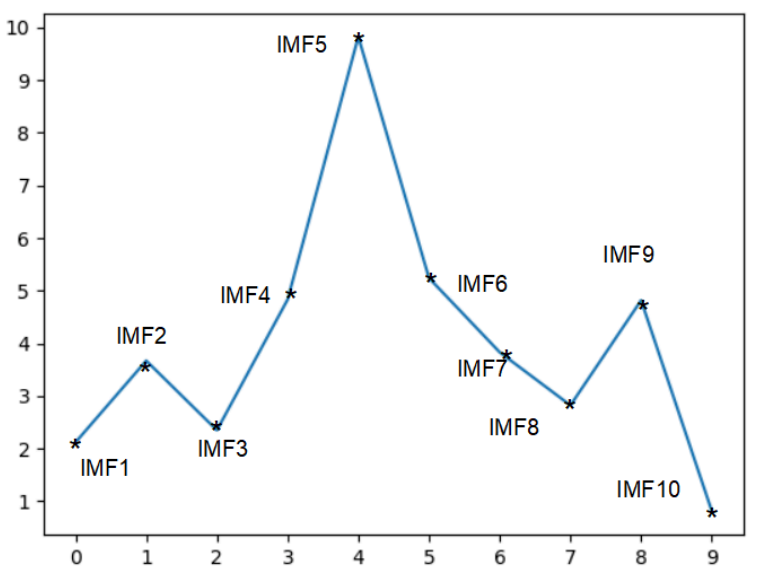

Figure 8: IMF component kurtosis

the effective IMF, which contains more vibration characteristic information generated by bearing fault.Then, the six IMF components with obvious fault characteristics are stacked into a multi-channel sample in the order of kurtosis from large to small, and the sample data dimension changes from $3000 \times 400$ to $3000 \times 6 \times 400$. The sample data dimension changes from $3000 \times 400$ to $3000 \times 6 \times 400$. Carry out the above operations on all sample signals, create the data set, and divide the data set into training set and test set. The final data set is shown in Table 2 .

\subsection{LSTM model parameter}

Design the LSTM according to the design principles described in Section 3.3, and adjust the relevant parameters through repeated tests. The final model structure and parameters are shown in Table 3.Units means the output length of the LSTM network. dropout means that the neuron in the network is set to zero with a certain probability $p$, which can suppress the over fitting problem to a

Table 2: Fault diagnosis training set and test set of rotating motor

\begin{tabular}{cccccc}
\hline Bearing state & Fault classification & Faulty diameter & Training set & Test set & Label \\
\hline normal & normal & - & 210 & 90 & 0 \\
Spur1 & Ball fault & 007 & 210 & 90 & 1 \\
Spur2 & Ball fault & 014 & 210 & 90 & 2 \\
Spur3 & Ball fault & 021 & 210 & 90 & 3 \\
Spur4 & Inner ring fault & 007 & 210 & 90 & 4 \\
Spur5 & Inner ring fault & 014 & 210 & 90 & 5 \\
Spur6 & Inner ring fault & 021 & 210 & 90 & 6 \\
Spur7 & Outer ring fault & 007 & 210 & 90 & 7 \\
Spur8 & Outer ring fault & 014 & 210 & 90 & 8 \\
Spur9 & Outer ring fault & 021 & 210 & 90 & 9 \\
\hline
\end{tabular}


Table 3: Main structural parameters of LSTM model

\begin{tabular}{cccc}
\hline Serial number & type & parameter & Other parameters \\
\hline 1 & Input Layer & Shape $=[3000,6,400]$ & \\
2 & LSTM Layer & $\mathrm{NU}=256, \mathrm{TS}=6$ & \\
3 & LSTM Layer & $\mathrm{NU}=256$ & Epoch $=50$ \\
4 & LSTM Layer & $\mathrm{NU}=128$ & Activation="relu" \\
5 & Full Connected Layer & $\mathrm{NU}=64$ & Classifer="softmax" \\
6 & Dropout Layer & Rate: 0.25 & Optimizer="AdamOptimizer" \\
7 & Output Layer & OC $=10$ & \\
\hline
\end{tabular}

Table 4: Test data accuracy under different super parameters

\begin{tabular}{|c|c|c|c|c|c|c|}
\hline \multicolumn{7}{|c|}{ Batch Size } \\
\hline & & 20 & 40 & 60 & 80 & 100 \\
\hline \multicolumn{7}{|c|}{ Accuracy date[\%] } \\
\hline \multirow[t]{5}{*}{ Learning rate } & 0.0005 & 99.8 & $\frac{L}{99.6}$ & 99.2 & 99.8 & 99.6 \\
\hline & 0.001 & 99.4 & 99.8 & 99.8 & 99.4 & 99 \\
\hline & 0.002 & 99.8 & 99.8 & 99.4 & 99 & 99.6 \\
\hline & 0.004 & 99.6 & 99.6 & 98.6 & 100 & 99.6 \\
\hline & 0.006 & 98.8 & 98.4 & 97.6 & 98.8 & 99.6 \\
\hline
\end{tabular}

certain extent, and improve the generalization performance of LSTM. The designed LSTM network is extracted by three LSTM feature extraction units, and a full connection layer for feature integration and classification through the layer. The output of LSTM in the first level feature extraction unit is $3 \times 256$, and then there are two feature extraction units, which realize the deep structure of the network and improve the expression ability of the network.

$\mathrm{NU}=$ number of units; $\mathrm{OC}=$ output channel;TS=time steps.

One of the important tasks of deep learning is to adjust the super parameters, as shown in Table 4. In this paper, the super parameters of batch size and learning rate are as follows. The mini batch gradient method is used to calculate the loss function. The batch size represents the number of samples processed in each batch, and the learning rate represents the update speed of weight and paranoid amount in back propagation.

The accuracy rate reflects the validity of the model. It can be seen from Table 4 that the accuracy rate of 80 batches and 0.004 learning rate is the highest, which can reach $100 \%$, and the average accuracy rate is $90 \%$.

\subsection{Results and analysis}

In order to illustrate the effectiveness of the method proposed in this paper, figure 9 compares the results of bearing condition monitoring realized by EEMD-LSTM method and BPNN, 1-DCNN [27], EMD-CNN [12] method.

Table 5 summarizes the results of bearing condition monitoring based on the same data set using the methods proposed in this paper, BPNN, 1-DCNN and EMD-CNN.Among them, BPNN structure is 2048-200-100-50-5, its activation function is ReLU, the learning rate of 1-DCNN method is 0.004, and the number of training is 500. EMD-CNN method will extract nine IMF components from the original data through EMD, and identify the fault through CNN.

In addition, in order to eliminate the influence of accidental error, each method has carried out 10 tests, taking the average value of various evaluation indexes as the test result, and the average value of 10 test results as the classification diagnosis performance evaluation index of this method.It can be seen that the diagnosis method based on EEMD and LSTM proposed in this paper is obviously superior to the traditional diagnosis method of BPNN.Compared with the advanced diagnosis methods 1-DCNN and EMD-CNN, the method proposed in this paper not only achieves $99.98 \%$ of the recognition indexes, which is better than 1-DCNN and EMD-CNN, but also has stable diagnosis results. The reason is that after EEMD processing, the signal-to-noise ratio of the signal is improved, and the effective IMF component with high kurtosis is constructed as the multi-channel input of LSTM..Through information 


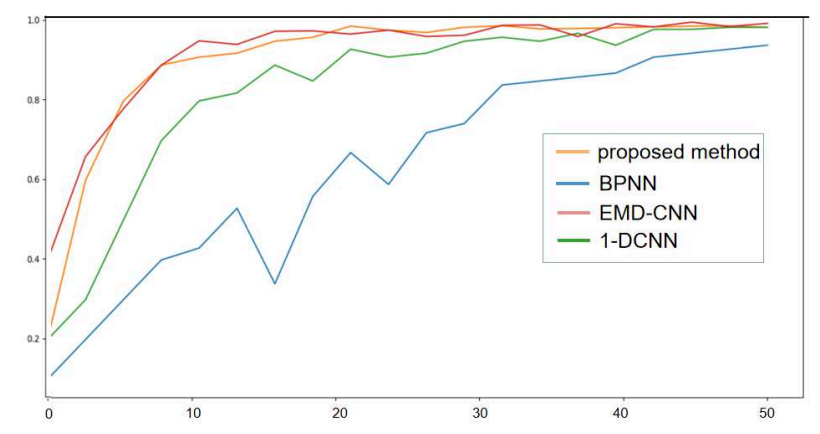

Figure 9: Change of fault diagnosis accuracy tested by different deep learning methods

Table 5: Statistics and general parameters of model test results

\begin{tabular}{|c|c|c|c|}
\hline Method & Accuracy\% & Recall\% & Common parameter \\
\hline EEMD-LSTM & 99.98 & 99.975 & $\begin{array}{l}\text { learning rate: } 0.004 \\
\text { tranning steps:50 } \\
\text { batch Size: } 80\end{array}$ \\
\hline BPNN & 68.73 & 69.66 & layers and cells: $2048-200-100-50-5$ \\
\hline 1-DCNN & 99.34 & 99.33 & $\begin{array}{l}\text { learning rate: } 0.004 \\
\text { tranning steps:500 }\end{array}$ \\
\hline EMD-CNN & 99.79 & 99.79 & 4 convolutions layers \\
\hline
\end{tabular}

fusion of LSTM, the weight of IMF component to the output is adaptively obtained.

\section{Conclusion}

In the first chapter, the paper reviews the machine learning methods in the field of fault diagnosis, points out the shortcomings of DNN method in the field of fault diagnosis, a fault diagnosis method based on ensemble empirical mode decomposition (EEMD) and long and short term memory neural network (LSTM) is proposed to solve the problem that the fault vibration signal of rotating machinery motor is usually nonstationary and the noise pollution is serious. It can realize the adaptive processing of data and simplify the operation of fault diagnosis. The paper introduces EEMD and LSTM methods in detail in the second and third chapter respectively, and puts forward the fault diagnosis process based on EEMD-LSTM in the fourth chapter, and in the fifth chapter Experimental verification was carried out. In this paper, The proposed method is compared with 1-DCNN and EMD-CNN.The experimental results show that:

(1) Combining the advantages of EEMD in analyzing and processing non-linear and non-stationary random signals and the powerful automatic feature extraction ability of LSTM, the intelligent bearing fault diagnosis is realized.

(2) Through kurtosis to determine the effective IMF component and build a more centralized data set with vibration information, the classification performance of LSTM network is improved.

(3) Through the test of test data, the proposed fault diagnosis method can achieve $99.98 \%$ accuracy, superior to the current advanced diagnosis methods 1-DCNN and EMD-CNN, with higher accuracy and better stability of diagnosis results.

Deep learning has a strong learning ability. It can update the weight by iterative learning method in the signal and automatically raising the fault feature. It has more advantages than those traditional artificial intelligence methods. What is learned from vibration signal and what is its physical meaning in each layer of deep learning? Although many literatures have done research and explanation in the field of image recognition, the relevant research in the field of fault diagnosis is not mature. The defects of long training time and poor generalization ability of LSTM have not been objectively described. The characteristics of LSTM in diagnosis need further study. Using the characteristics of vibration signal to design a more "suitable" deep learning model will be the direction of future research [20] [23].In addition to the application in the field of industrial robots, fault diagnosis can also be derived 
and applied to the fields of transportation [11], finance [11] and supply chain [9] [8], which has a broad application prospect.

\section{Funding}

The research was funded by the National Key R\&D Program of China, (No.2018YFB1702700).

\section{Author contributions}

The authors contributed equally to this work.

\section{Conflict of interest}

The authors declare no conflict of interest.

\section{References}

[1] Alangari, H.; Kimura, Y. (2017). A Hybrid EMD-Kurtosis Method for Estimating Fetal Heart Rate from Continuous Doppler Signals, physiology, 8, 2017.

[2] Andrychowicz, M.; Denil, M. (2016). Learning to learn by gradient descent by gradient descent, Proc. NIPS, 2016.

[3] Auli, M.; Galley, M.; Quirk, C.; Zweig, G. (2013). Joint Language and Translation Modeling with Recurrent Neural Networks, Proc. of EMNLP, 2013.

[4] Bahdanau, D.; Cho, K.; Bengio, Y. (2014). Neural machine translation by jointly learning to align and translate, arXiv preprint arXiv, 1409.0473, 2014.

[5] Bengio, Y. (2009). Learning deep architectures for ai, Found, Trends Mach. Learn, 2(1), 1-127, 2009.

[6] Bengio, Y.; Courville, A.; Vincent, P.(2013). Representation learning: a review and new perspectives, IEEE Trans. Pattern Anal. Mach. Intell, 35(8), 1798-1828, 2013.

[7] Blitzer, J.; McDonald, R.; Pereira, F. (2006). Domain adaptation with structural correspondence learning, Proceedings of EMNLP, 120-128, 2006.

[8] Chu, X.; Liu, J.; Gong, D.; Wang, R. (2019). Preserving Location Privacy in Spatial Crowdsourcing under Quality Control, IEEE Access, 7, 155851-155859, 2019.

[9] Chu, X.; Zhong, Q.; Li, X. (2018). Reverse channel selection decisions with a joint third-party recycler, International Journal of Production Research, 56(18), 5969-5981, 2018.

[10] Gong, D.; Tang, M.; Liu, S.; Xue, G.; Wang, L.(2019). Achieving sustainable transport through resource scheduling: A case study for electric vehicle charging stations, Advances in Production Engineering \& Management, 14(1), 65-79, 2019.

[11] Gong, D.; Liu, S.; Liu, J.; Ren, L.(2019). Who benefits from online financing? A sharing economy E-tailing platform perspective, International Journal of Production Economics, DOI: 10.1016/j.ijpe.2019.09.011.

[12] Gu, N.L.; , Pan, H. (2017). Bearing Fault Diagnosis Method Based on EMD-CNNs, CSMA, 2017.

[13] He, Q.; Li, P.; Kong, F. (2012). Rolling bearing localized defect evaluation by multiscale signature via empirical mode decomposition, J. Vib. Acoust, 134, 061013, 2012.

[14] Hinton, G.E.; Salakhutdinov, R.R.(2006). Reducing the dimensionality of data with neural networks, Science, 313(5786), 504-507, 2006. 
[15] Huang, N.E.; Shen, Z.; Long, S.; Wu, M.; Shih, H.; Zheng, Q.; Yen, N.-C.; Tung, C.; Liu, H. (1998). The empirical mode decomposition and the Hilbert spectrum for nonlinear and nonstationary time series analysis, Proc. R. Soc. A Math. Phys. Eng. Sci, 454, 903-995, 1998.

[16] Karpathy, A.; Fei-Fei, L. (2015). Deep visual-semantic alignments for generating image descriptions, Proceedings of the IEEE Conference on Computer Vision and Pattern Recognition, 3128-3137, 2015.

[17] Lei, Y.; He, Z.(2009). Application of the EEMD method to rotor fault diagnosis of rotating machinery, Mechanical Systems and Signal Processing, 23(4), 1327-1338, 2009.

[18] Lei, Y.; He, Z. (2011). EEMD method and WNN for fault diagnosis of locomotive roller bearings, Expert Systems with Applications, 38(6), 7334-7341, 2011.

[19] Liu, R.; Yang, B. (2018). Artificial intelligence for fault diagnosis of rotating machinery, Mech. Syst. Signal Process, 135, 33-47, 2018.

[20] Nazifa, T.S.; Mohaed, S.F.; Amin, A.B. (2019). A Brief Discussion on Supply Chain Management in Construction Industry, Journal of System and Management Science, 9(1), 69-86, 2019.

[21] Randall, R.B.; Antoni, J.(2011). Rolling element bearing diagnostics-A tutorial, Mech. Syst. Signal Process, 25, 485-520, 2011.

[22] Song, L.; Wang, H.; Chen, P. (2018). Vibration-Based Intelligent Fault Diagnosis for Roller Bearings in Low-Speed Rotating Machinery, IEEE Trans. Instrum. Meas, 67, 1887-1899, 2018.

[23] Tabsh, Y.; Davidaviciene, V. (2016). Information and Communication Technologies in Energy Management, Journal of System and Management Science, 6(4), 67-81, 2016.

[24] Wang, D.; Tsui, K. (2018). Brownian motion with adaptive drift for remaining useful life prediction: revisited, Mech. Syst. Signal Process, 99, 691-701, 2018.

[25] Wang, J.; Du, G. (2020). Fault diagnosis of rotating machines based on the EMD manifold, Mech. Syst. Signal Process, 135, 106443, 2020.

[26] Wang, T.; Zhang, M. (2012). Comparing the applications of EMD and EEMD on time-frequency analysis of seismic signal, Journal of Applied Geophysics, 83, 29-34, 2012.

[27] Wu, C.; Jiang, P. (2019). Intelligent fault diagnosis of rotating machinery based on onedimensional convolutional neural network, Computers in Industry, 108, 53-61, 2019.

[28] Wu, Z.; Huang, N.E. (2006). Ensemble empirical mode decomposition:a noise assisteted data analysis method, Advances in Adaptive Data Analysis, 1(1), 1-41, 2009.

[29] Xu, X.; Chen, R. (2007). Recurrent Neural Network Based On-line Fault Diagnosis Approach for Power Electronic Devices, ICNC, 24-27, 2007.

[30] Yink, W.; Kann, K.(2017). Comparative Study of CNN and RNN for Natural Language Processing, Computer Science, 1702.01923, 2017.

[31] Zhao, H.; Sun, S. (2016). Sequential Fault Diagnosis based on LSTM Neural Network, IEEE Access, 6, 12929-12939, 2018.

[32] Zhao, R.; Wang, J.; Yan, R.; Mao, K. (2016). Machine health monitoring with LSTM networks, Proceedings of the 2016 10th International Conference on Sensing Technology (ICST), 1-6, 2016.

[33] [Online]. Available: www.phmsociety.org/competition/PHM/09, Accesed on 14 February 2019. 


\section{(c) (i) (8)}

Copyright (C)2020 by the authors. Licensee Agora University, Oradea, Romania.

This is an open access article distributed under the terms and conditions of the Creative Commons Attribution-NonCommercial 4.0 International License.

Journal's webpage: http://univagora.ro/jour/index.php/ijccc/

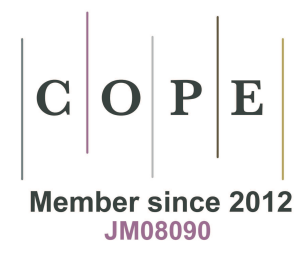

This journal is a member of, and subscribes to the principles of, the Committee on Publication Ethics (COPE).

https://publicationethics.org/members/international-journal-computers-communications-and-control

Cite this paper as:

Zou, P.; Hou, B.; Jiang, L.; Zhang, Z. (2020). Bearing Fault Diagnosis Method Based on EEMD and LSTM, International Journal of Computers Communications \& Control, 15(1), 1010, 2020. https://doi.org/10.15837/ijccc.2020.1.3780 